\title{
LIFT- A Linear Two-Phase Commit Protocol
}

\author{
Aman Kumar Pandey \\ Dept. of Computer Sc. \& Engg. \\ M.M.M University of Technology \\ Gorakhpur- 273010, India \\ amanpnd60@outlook.com
}

\author{
Sarvesh Pandey \\ Dept. of Computer Sc. \& Engg. \\ M.M.M University of Technology \\ Gorakhpur- 273010, India \\ pandeysarvesh100@gmail.com
}

\author{
Udai Shanker \\ Dept. of Computer Sc. \& Engg. \\ M.M.M University of Technology \\ Gorakhpur- 273010, India \\ udaigkp@gmail.com
}

\begin{abstract}
-
Two phase commit (2PC) protocol is used to coordinate transaction commitment in distributed database system. The $2 \mathrm{PC}$ protocol is further classified into linear and centralized versions. The linear version of $2 \mathrm{PC}$ i.e. L-2PC protocol runs serially whereas in the centralized version, the commit process allows every site involved in executing the transaction to prepare and commit in parallel. In past, very little efforts have been made to overcome the unfavourable message overhead, poor recovery process and some other problems associated with L-2PC protocol. This paper proposes a Linear and Fast-paced recovery centred Transaction commit (LIFT) protocol to improve the existing recovery mechanism of linear $2 \mathrm{PC}$ by allowing autonomy to the participating cohorts to inform the cohort-in-doubt about the distributed transaction's state instead of always looking for coordinator's stand. The performance results confirm that proposed protocol will be a better alternative.
\end{abstract}

Keywords-Linear 2PC protocol, LIFT protocol, Recovery mechanism, Transaction processing

\section{INTRODUCTION}

Transaction composed of set of data operations i.e. read and write, is a logical work unit [1] [2]. It must follow ACID (Atomicity, Consistency, Isolation and Durability) property [3] [4]. The atomicity refers to all or none transaction behaviour. It is required to ensure that either each one or not any of the operations belonging to a transaction is executed. The second important property is consistency which is nothing but the correct transaction code from SQL designer's point of view; concurrency control (CC) protocols are required to put in place to ensure the same in case of concurrent transaction execution. The third pillar of transaction management is isolation. In isolation, each transaction must be executed without knowing what is happening with other transactions. The last important property is durability which ensures all updates done by a transaction must become permanent. Recovery manager are responsible for the transaction's durability.

Two phase commit (2PC) protocol works in two stages — voting phase \& decision phase [5] [6]. The distributed version of $2 \mathrm{PC}$ is responsible for atomic commitment of any transaction in distributed environment. The objective of the first phase is to reach a common decision and second phase is to execute the decision. It coordinates every process that participates in executing the distributed transaction, and to eventually reach at a unanimous decision on whether to abort or commit. Blocking nature of $2 \mathrm{PC}$ protocol puts an adverse effect on transaction processing applications. The main reason of such problem is due to failure of the coordinator and/or cohorts at an intermediate stage. The problem may arise when cohorts send the PREPARED message to their coordinator and wait for the final decision from coordinator site. At the same time, the coordinator is unable to communicate the global decision message to cohorts due to failure. Transaction gets blocked and its participating cohorts will keep on holding resources that they have already locked before initiating the commit process until coordinator's recovery. Because of the failure of coordinator, blocked transaction diminishes the availability of the entire system.

In broad sense, the commit protocol can be classified as following: Centralized 2PC and Linear 2PC (L-2PC) [1] [7]. The most used variant of $2 \mathrm{PC}$ is Centralized $2 \mathrm{PC} /$ Classical 2PC/ 2PC Protocol. The centralized 2PC assigns the role of the coordinator to the site of the origin of the transaction. Centralized control of the commit process in 2PC eventually allows every site of the transaction to PREPARE and COMMIT in parallel. Fig. 1 clearly explains the working of Centralized 2PC protocol through a diagrammatic representation.

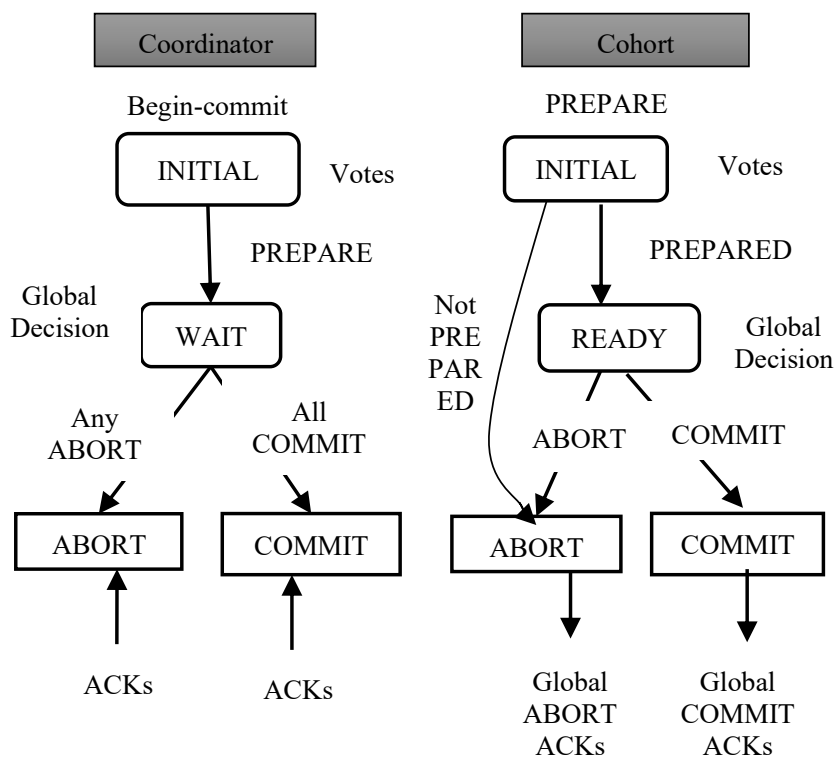

Fig 1: Working of Centralized 2PC Protocol 
The 2PC protocol, blocking by nature, was first analysed using finite state automata (FSA) in [1]. To avoid such blocking nature of $2 \mathrm{PC}$, an ample research studies has been done in past [8]. Non-blocking refers to the situation where operational sites may continue their transaction processing tasks even when failure occurs at some of its sites [9] [10]. Centralized version of $2 \mathrm{PC}$ was further extended with the presumed optimization strategy [11] [12] [13] i.e. presumed abort $(\mathrm{PrAb})$ protocol and presumed commit (PrCo) protocol [14]. The concept of presumed optimization strategy is further explored by the $\mathrm{AP}^{3}$, an adaptive participant's presumption protocol. $\mathrm{AP}^{3}$ protocol leverages the performance advantages of 1-phase commit (1PC) protocol and wide acceptability of $2 \mathrm{PC}$ protocols [15] [16]. For the commitment process, $\mathrm{AP}^{3}$ works as a $1 \mathrm{PC}$ protocol only when the validation of deferred consistency constraints is not imposed on the transaction. In all the other cases, the $\mathrm{AP}^{3}$ protocol dynamically shifts to the $2 \mathrm{PC}$ protocol. Hence, the applicability and performance of the original $\mathrm{AP}^{3}$ protocol is expected to make it a good pick in designing of upcoming distributed database system (DDBS). The core of the intelligent $\mathrm{AP}^{3}$ is to further extend the basic $\mathrm{AP}^{3}$ in two important directions like applicability and efficiency. To deal with this blocking problem, two new non-blocking protocols were proposed; the first one is three phase central site protocol and the second is three phase distributed commit protocol. Basically, three phase commit (3PC) protocol was derived from 2PC protocol with an extra "PREPARE TO COMMIT" state [17] [18] [19]. This was the least modification that can be made to $2 \mathrm{PC}$ protocol in order to satisfy the theorem of non-blocking. Termination protocol then presented to be invoked when coordinator failure occurs in a centralized commit protocol or any site failure occurs in decentralized commit protocol. Even though the 3PC protocol was non-blocking but it was not efficient because of one extra round of message overhead.

The 2PC is further extended and an Easy-Commit protocol is proposed. It is claimed that the performance is improved through swapping of the communication order [20] i.e. instead of writing in the stable storage first and then sending the message to other sites, the above order is reversed. It does so without adding any extra site for exhibiting non-blocking behaviour. Easy Commit protocol required each site, either coordinator site or cohorts, to communicate the global decision to all other sites before they committed the transaction. They introduced multiple entries in the stable storage to ease the recovery process during the site failures. The message complexity of Easy Commit protocol is $\mathrm{O}\left(\mathrm{n}^{2}\right)$ which is greater than the $2 \mathrm{PC}$ protocol message complexity of $\mathrm{O}(\mathrm{n})$ [20]. Easy Commit protocol is non-blocking but 2PC protocol still has the lower message complexity. The message complexity of $3 \mathrm{PC}$ protocol is $\mathrm{O}(\mathrm{n})$ which is also less than Easy Commit protocol.
However, Easy Commit protocol is efficient version of $3 \mathrm{PC}$ protocol.

The L-2PC Protocol is another variant of 2PC. In L-2PC, there is a sequential flow of message communication in commit processing. Let us take an example to explain the working of L-2PC. Suppose, a transaction is initiated at site $\mathrm{A}$, and it requires data items to be accessed from site $\mathrm{A}$, site B, and also from site C. So, after completion of execution at all its participating sites, transaction, which is being originated at site $\mathrm{A}$, will further initiates its commit processing by sending PREPARE message in serial order i.e. form site A to site $\mathrm{B}$, then site $\mathrm{B}$ to site $\mathrm{C}$. Further site $\mathrm{C}$ (last site) acts as the coordinator site which notifies the abort or commit decision to its predecessor site.

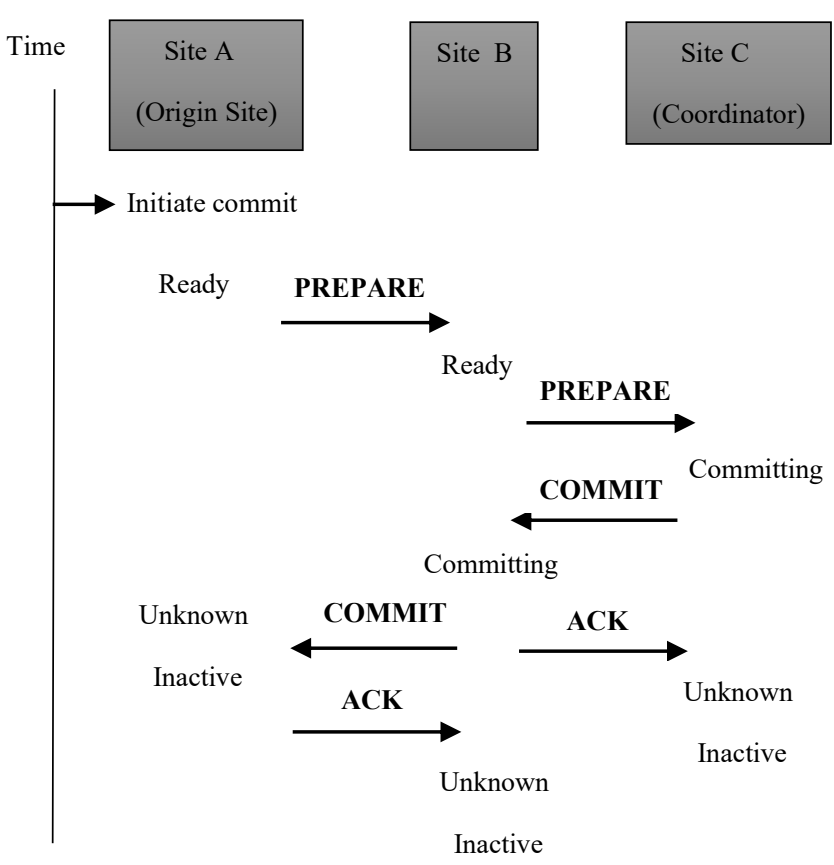

Fig 2: Timeline Diagram of Linear Two-Phase Commit Protocol

\section{First Phase}

The commit procedure begins when the site of origin decides to initiate the commit. The site having received answer to all its work requests has a complete list of sites used by the transaction.

1. Site of origin writes the status of READY in log and sends the PREPARE message (PREPARE message contains the transaction id and the sites used list with the site of origin at the head of the list) to the next site in the sites used list. The selection of next site is depending upon which site is present at the top of the site list. 
2. After receipt of PREPARE message from the site of origin, site $B$ again writes the status of READ in its log and posts the PREPARE message to next site.

\section{Second Phase}

The last site in L-2PC protocol works as a coordinator site which is an authority to send back the commit or abort decision to the predecessor site.

1. When site $\mathrm{C}$ receives the 'PREPARE' message from its predecessor site, it simply writes the status of 'COMMITTING' in its log and posts the COMMIT message to its predecessor site.

2. After getting the 'COMMIT' message from the last site (coordinator), the site $\mathrm{B}$ writes the status of 'COMMITTING' in its log and sends the ack message to its successor and 'COMMIT' message to its predecessor simultaneously.

3. When the 'COMMIT' message is received at the site of origin, it sends the ack message to corresponding successor site i.e. site B

When the COMMIT message is acknowledged, the site enters the UNKNOWN state by logging done, dropping the transaction descriptor from volatile memory and forgetting about the transaction. The timeline diagram of the $\mathrm{L}-2 \mathrm{PC}$ protocol is presented in Fig. 2. The commit procedure starts when site A (origin site) decides to initiate the commit. The origin site has a complete list of the sites used by the transaction. Each site, starting with the site of origin, enters in the READY state and then notifies the next site is the list to prepare for commit.

The two protocols described above have their own advantages and disadvantages. The L-2PC protocol always needs fewer messages and faster execution of the commit processing [7]. At the same time, centralized 2PC ensures fewer message delays when three or more sites are involved in commit processing. Here, instead of propagating the PREPARE and COMMIT messages in serial i.e. from site to site, a single site can be chosen to send the PREPARE and COMMIT message in parallel [21]. Moreover, communication is done through the central site known as coordinator, and thus there is no direct communication between the cohorts. The coordinator is accountable for sending the PREPARE message to every cohorts and when all the cohort votes are received, the central coordinator site determines to either ABORT or COMMIT the transaction [20] [22]. Table 1 represents comparison of linear and centralized versions of commit protocol based on various parameters like total messages, message delays, message delay to commit point, recoverable state changes and readonly sites.

\begin{tabular}{|c|c|c|}
\hline & Linear & Centralized \\
\hline $\begin{array}{l}\text { Total } \\
\text { Messages }\end{array}$ & $3(N-1)$ & $4(\mathrm{~N}-1)$ \\
\hline $\begin{array}{l}\text { Message } \\
\text { Delays }\end{array}$ & $2 \mathrm{~N}-1$ & 4 \\
\hline $\begin{array}{l}\text { Message Delays } \\
\text { to commit Point }\end{array}$ & $\mathrm{N}-1$ & 2 \\
\hline $\begin{array}{l}\text { Recoverable State } \\
\text { Changes }\end{array}$ & $2 \mathrm{~N}$ & $2 \mathrm{~N}$ \\
\hline $\begin{array}{l}\text { Read-only } \\
\text { sites }\end{array}$ & No help & $\begin{array}{l}-2 \text { messages } \\
\text { per read-only }\end{array}$ \\
\hline
\end{tabular}

Table 1: Comparison of Linear and Centralized Commit

To resolve the problem, a new protocol LIFT is proposed. Here, each site in first phase, will receive a 'Prepare Ack' message from its successor site on sending 'PREPARE' message. This helps in dealing with timeouts of cohorts.

The organization of the remaining portion of this technical paper is as follows: The DDBS model is presented in section II. In section III, the LIFT protocol is discussed in detail. Finally, section IV concludes the work and represents directions for the future research.

\section{SYSTEM MODEL}

The commit processing and real-time scheduling algorithms are the important research directions in DDBS [23]. A lot of advancements in classical 2PC are proposed in past in context of time constrained databased but comparatively less efforts are made in context of non-realtime DDBS [24][25][26][27][28][29][30][31]. The simple DDBS model ensuring atomic commitment of distributed transactions is shown in Fig 3. The above model is simply an extension to the conventional database system model described in [6]. The loosely coupled distributed database is generally composed of a finite number of interconnected data servers. A server may consist of main memory and disk and is equipped with one CPU. It can utilize non-volatile storage and also redundancy for the purpose of creating stable storage [32]. Stable storage is both reliable and nonvolatile. Servers are non-sharable in terms of storage and 
communicate with the help of messages. Each server stores and manages a small part of the database called an individual concrete database. An assumption has been made that all the concrete databases and their interconnections to server is known to all servers. The DDBS contains the set of data objects; smallest unit of data to be accessed. Every data object one is stored at only one site. The transaction contains a sequence (patterns) of operations to be done on data items.

Some of the components of our proposed model are as follows: (i). Transaction Manager (TrM), (ii). Resource Manager (ReM), and (iii). Data Operation Scheduler (DaOS) [17]. The TrM is authenticated for originating lock requests, DaOS schedules all such requests based on the terms of CC scheme employed, and DaM is authenticated for allowing access to data requests.

The Database Application generates transactions. The TM makes appropriate lock requests based on possible data item access list to execute the given transaction. The scheduler dynamically schedules the parallel transaction execution producing a schedule as well as the parallel execution of non-database operations with database operations. The data manager executes partial operation of transactions. In proposed protocol, a user can perform only read and write operations inside transactions and locks can be permitted and freed only by the scheduler. Locking strategy is used to avoid conflict between transactions.

The ReM provides services of the I/O for modifying data and services of CPU for processing the data items. The scheduling of CPU and I/O both are performed using the queues available at the $\mathrm{CPU}$ and $\mathrm{I} \backslash \mathrm{O}$ respectively. The cohorts are ordered in these queues based on their priorities. The CPU is scheduled using preemptive high priority scheme. This scheme ensures that higher priority cohort from $\mathrm{CC}$ queue can preempt the lower priority cohort, and the blocked cohort may resume its execution only if its priority becomes highest among all the cohorts in the queue or if the CPU queue becomes empty. In simple terms, the CPU is allocated to cohorts based on their priorities.

The transaction execution must be atomic such that either it commits through making its effects permanent or aborts without leaving any effect at all. Moreover, transactions are serializable in such a way that their overall effect is as if they had been run by following an order in sequential way. There are $\mathrm{N}$ number of transactions $\mathrm{T}_{1}, \mathrm{~T}_{2}, \mathrm{~T}_{3}, \ldots, \mathrm{T}_{\mathrm{N}}$ participating in the commit processing. The transaction processing involves $N$ number of sites $S_{1}, S_{2}, S_{3}, S_{N}$ arranged serially. Site $S_{1}$ is the origin site where the last site $S_{N}$ becomes the coordinator site in the transaction processing. The flow of message in the first phase is in forward direction serially and the message flow in the second phase is in backward direction serially [33].

Site $\mathrm{S}_{\mathrm{N}}$ is the successor of site $\mathrm{S}_{(\mathrm{N}-1)}$ and site $\mathrm{S}_{(\mathrm{N}-1)}$ is the predecessor of site $S_{N}$. The ACK message is introduced in the second phase when Site $\mathrm{S}_{(\mathrm{N}-1)}$ receives the commit message from site $\mathrm{S}_{\mathrm{N}}$ and acknowledges the commit message sent by site $\mathrm{S}_{\mathrm{N}}$. The data items are stored at operational sites linked through underlying network. Every site comprises DaM and TrM. The DaM handles the individual database and the TrM monitors the processing of transaction. The data item is updated by the DaM and creates a $\log$ for the update and sends the replies back to the coordinator's site.

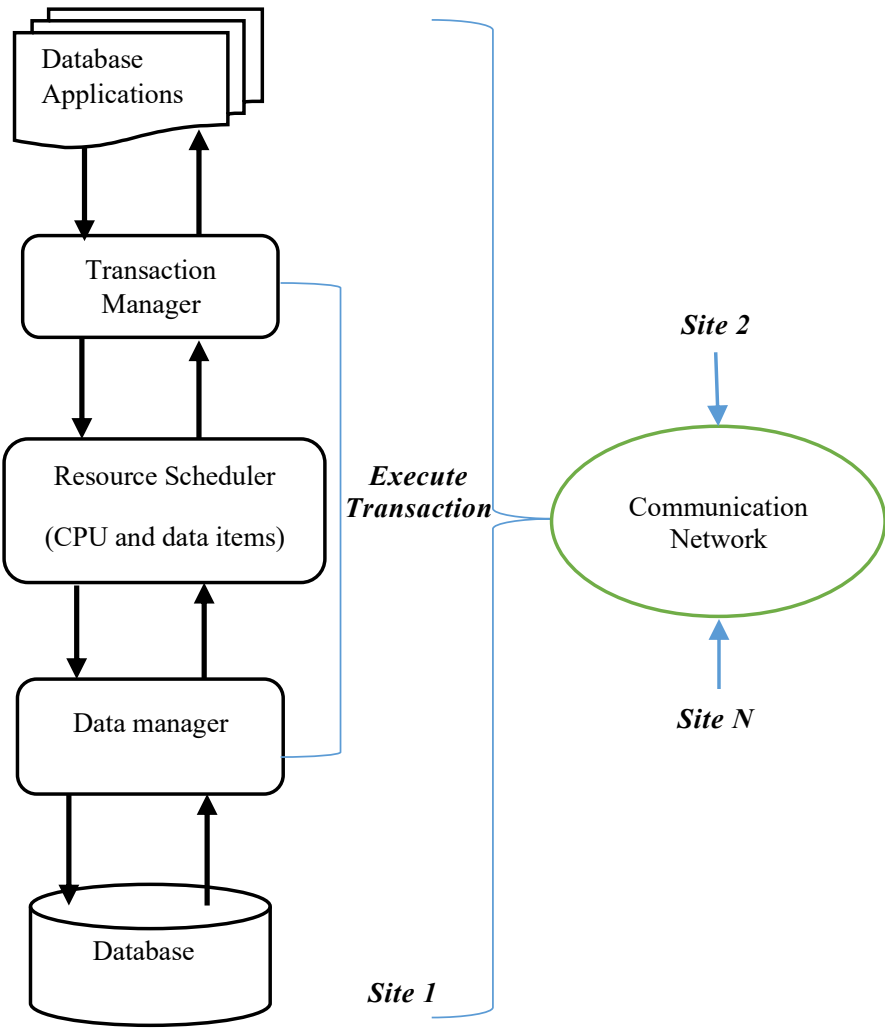

Fig 3: Conventional DDBS Model

Our assumption is that the network is susceptible to site failure and link failure. In case of failure of any operational site, it gets blocked and other sites identify this piece of information. We also undertake that the network partitioning can arise in the commit processing. When there is a failure because of partitioning of the network then the operational sites are branched into two or more clusters in which two sites in same cluster can establish a communication with each other but if sites are in different group then they cannot communicate. The communication medium gives the facility to transfer the message between operational sites. 
Suppose the site $S_{1}$ wants to send the message to site $S_{2}$ then message is handed over to the communication medium responsible for delivering of the message from source site $S_{1}$ to destination site $S_{2}$ in a finite amount of time. For any pair of sites $S_{1}$ and $S_{2}$, the communication medium sends the message to $S_{2}$ in the same order that site $S_{1}$ handed over the message to the medium. It is necessary that servers may be able to forget about the transaction after small amount of time. A server forgets about the transaction (control information for the transaction) by removing its $\log$ from volatile storage.

\section{LIFT: A LINEAR TWO-PHASE COMMIT PROTOCOL}

The L-2PC protocol suffers from the problem of restarting the distributed transaction's entire commit procedure in case of timeout event with any of its participating cohort. Here, the flow of message is always unidirectional - forward in first phase, and backward in second phase. This eventually adds up in making the recovery procedure more complex and inefficient, and becomes one of the major causes for degraded system performance. To address above problem, the Linear and Fast-paced recovery centred Transaction commit (LIFT) protocol is proposed that guarantees the fast recovery in case of failure.

To understand the LIFT protocol, let us take an example scenario. Suppose that there are three sites, named site $\mathrm{A}, \mathrm{B}$ and $\mathrm{C}$, engaged in the execution of a given distributed transaction. The first site is the origin site - site A in this case, and the last site acts as the coordinator - site $\mathrm{C}$ in this case. Similar to L-2PC, the LIFT protocol also works in two steps or phases.

The first one LIFT protocol phase is diagrammatically represented in Fig 4. The origin site (site A) initiates the commit process by writing status of 'Ready' in the log and communicating the 'Prepare' message to next site (site B). The selection of next cohort is based on the current cohort executing from the 'execution wise-ordered list' of cohorts that origin site (site A) sends to its immediate next site piggybacked with 'Prepare' message, which then sends to its immediate next site in a similar fashion, and so on. After receiving the 'Prepare' message, site B must check to see whether the indicated transaction is active or not. If indicated transaction is active, site $\mathrm{B}$ acknowledges the 'Prepare' message sent by origin site A by sending back the 'Prepare Ack' message to it. Otherwise, ABORT the transaction. After acknowledging the 'Prepare' message, site B writes the status of 'Ready' in the log and sends the 'Prepare' message to next site which is $\mathrm{C}$ in our case. When site $\mathrm{C}$ receives the 'Prepare' message, it again checks whether the indicated transaction is still active or not. If indicated transaction is active, it acknowledges the 'Prepare' message sent by site B by sending back the 'Prepare Ack' message to it. Since, site $\mathrm{C}$ is the last site to be visited during commit processing, it works as the coordinator site. It is, therefore, responsible for sending the COMMIT or ABORT decision to its predecessor site in serial fashion.

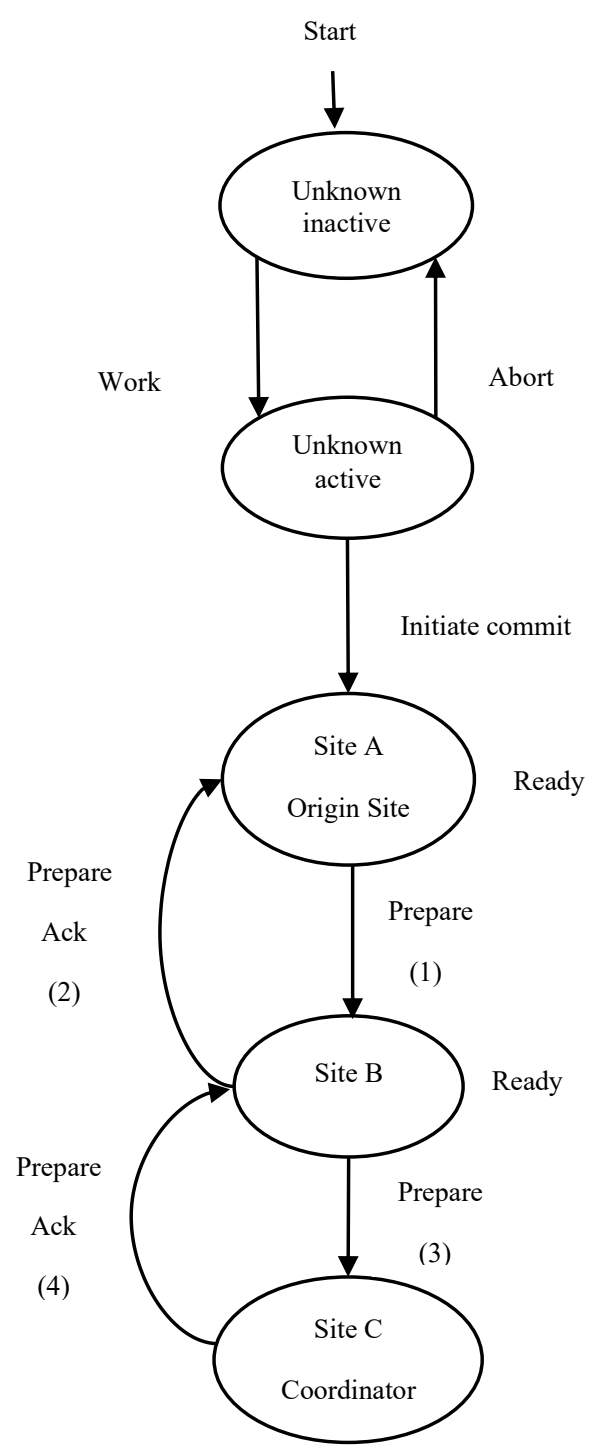

Fig 4: First Phase of LIFT Protocol

The second one LIFT protocol phase is diagrammatically represented in Fig 5. Second phase is initiated if the given transaction is active at the last site and no other condition prevents to commit. The last site gives the final decision i.e. either COMMIT or ABORT to its predecessor site B. Here, the last site i.e. site $\mathrm{C}$ enters in the COMMITTING state by writing the status of COMMITTING in its $\log$ and communicating the COMMIT message to its 'Ready' predecessor. Each 'Ready' site depends upon its successor to respond to its 'Prepare' message request with either ABORT or COMMIT decision. 
Upon receipt of COMMIT message, a participating site enters in status of COMMITTING and sends following two messages in parallel.

1. It acknowledges the COMMIT message to its successor by sending back the 'Commit Ack' message to it.

2. It sends the COMMIT message to its READY predecessor.

Each COMMITTING site must maintain its volatile memory transaction descriptor until the COMMIT message is acknowledged. When origin site (site A) receives the COMMIT message from its immediate successor (site B), it writes the status of COMMITTING in log and acknowledges the COMMIT message. After this, it returns to an UNKNOWN state by dropping all volatile memory records of the transaction. ABORT message propagates in the same way as COMMIT.

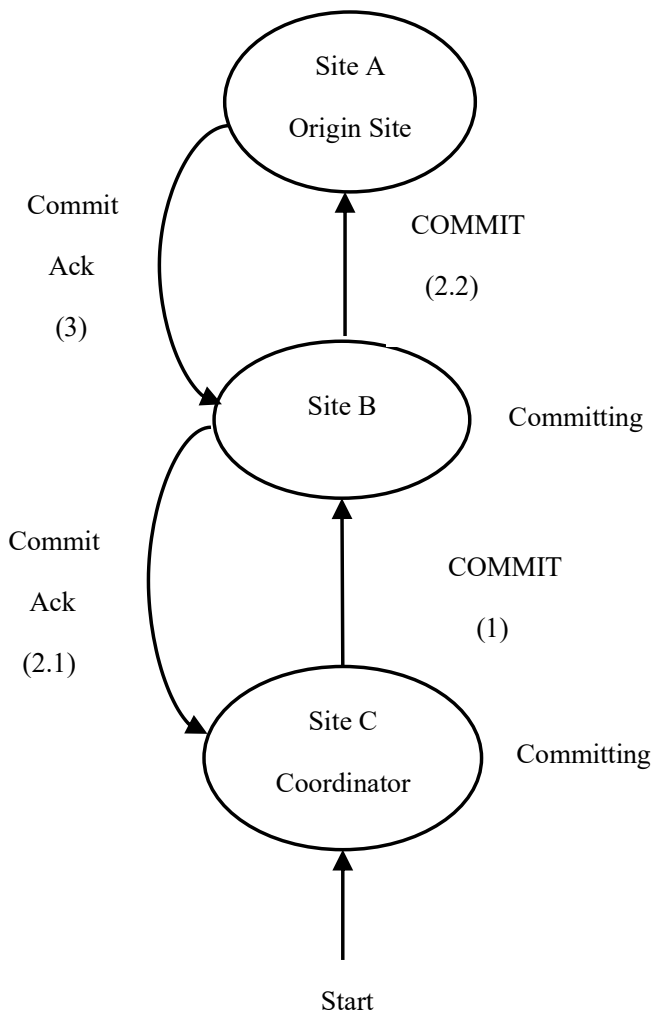

Fig 5: Second Phase of LIFT Protocol

In both the state diagrams i.e. Fig. 4 and Fig. 5 representing the 2 phases of LIFT protocol respectively, the message flow sequence is represented with number for better clarity. The pseudo code of the protocol proposed is presented below.

\section{LIFT ALGORITHM:}

Site A (Site of origin):

1. Write status of Ready in log;

2. Communicate PREPARE message (transaction id + sites used list with site of origin at head of list) to next site;

Activate time-out;

Site B:

1. Wait for PREPARE message from site of origin;

2. After getting PREPARE message, site B sends back 'Prepare Ack' message to its predecessor site;

3. When PREPARE message is received at site $B$ then Begin

Site B must check to see whether indicated transaction is 'active' at site and then write status of Ready in log;

Communicate PREPARE message to next site;

end

else

begin

PREPARE message is acknowledged with an Abort message to sender; end;

Site C (Coordinator site):

The last site acts as a coordinator,

1. Wait for PREPARE message from predecessor site $\mathrm{B}$;

2. After receiving PREPARE message, coordinator site C sends back 'Prepare Ack' message to its predecessor site and writes status of COMMITTING in log;

3. Communicate COMMIT message to its predecessor site i.e. site B;

Site B:

1. Wait for COMMIT message from site $\mathrm{C}$;

2. After getting COMMIT message, site $\mathrm{B}$ writes status of COMMITTING in log and communicates following message simultaneously;

i) Site B sends back ack message to its successor site $\mathrm{C}$;

ii) And, site $\mathrm{B}$ sends COMMIT message to its predecessor site i.e. site of origin;

\section{Site A (Site of origin):}

1. After receiving COMMIT message from site $\mathrm{B}$, it sends back ack message to its successor site B;

2. After COMMIT is acknowledged, site enters in UNKNOWN states by logging done and 'forgetting' the transaction;

In brief, it may be said that poor recovery process of L2PC protocol is made efficient by giving autonomy to the participating cohorts to inform the cohort-in-doubt about the distributed transaction's state instead of always looking for coordinator's stand. 


\section{SIMULATION STUDY AND PERFORMANCE RESULTS}

A DDBS comprising 12 sites is computer-generated through simulation. Each site consists of 2000 data items. Further, each global transaction comprises 3 cohorts. The transaction may request for 4 to 20 data items during its execution lifetime. For number of data item requests by a transaction, uniform distribution is followed. The probability of write operations is assumed to be 0.6 . For each transaction-related message, communication delay is 1 millisecond. The delay incurred in accessing disk is assumed to be 5 milliseconds consisting of rotational delay and the data transfer time. During simulation, the performance metric considered is throughput of the system. Some more assumptions considered during this simulations study are as following.

- We have employed the closed queuing standard wherein Poisson distribution is utilized for generating transactions.

- The TrM manages the execution of transaction. Also, whenever transaction is aborted no matter for whatsoever reason, it is automatically re-submitted for execution until it eventually reaches at final decision.

- The hard disk is utilized as a stable storage; no primary memory is used.

- Strict two phase locking (Strict 2PL) is employed as a $\mathrm{CC}$ protocol.

- The database considered is a logical collection of data. Locking granularity is assumed to be at data item level.

- When some transaction requests for a lock then the distributed deadlock detection algorithm is utilized to identify whether it may result into the deadlock or not. In case it is found that deadlock may occur on granting the lock then lock is not granted.

- The transaction log is comprised of pages; a page can be modified (read/write) only once by any transaction.

We have evaluated the proposed LIFT protocol's performance against that of $2 \mathrm{PC}$ protocol's performance. The performance metric considered for the assessment is throughput.

Tranasction Arrival Rate vs Throughput

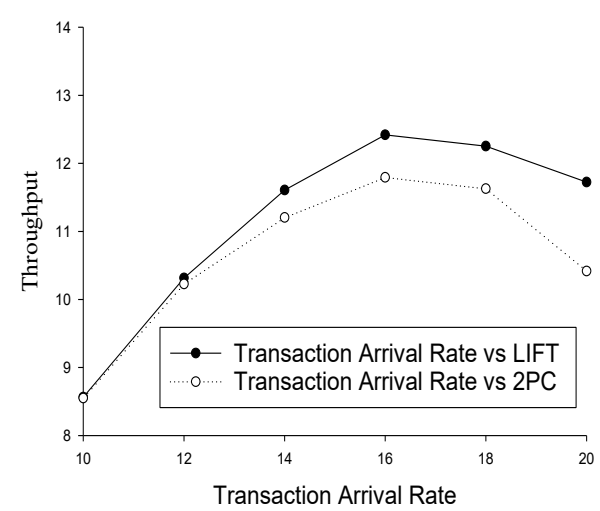

Fig 6: Comparing LIFT with 2PC using 'throughput' metric: No failure
Fig. 6 shows the throughput of the system under $2 \mathrm{PC}$ and LIFT with varying transaction arrival rate. It may be clearly interpreted that the proposed LIFT protocol ensures marginally higher throughput compared with L-2PC. The marginal improvement shown with LIFT is only due to its serial fashion of executing the commit procedure [7].

Fig. 7 shows the performance of LIFT protocol with failures. As evident from Fig. 7, the LIFT protocol's performance is considerably improved than that of 2PC. It is mainly because of the duplex message communication between participants of distributed transaction during commit processing. This help, in terms of system throughput, due to providing somewhat more information to the cohorts that virtually acts as an internal node during their serial order commit processing.

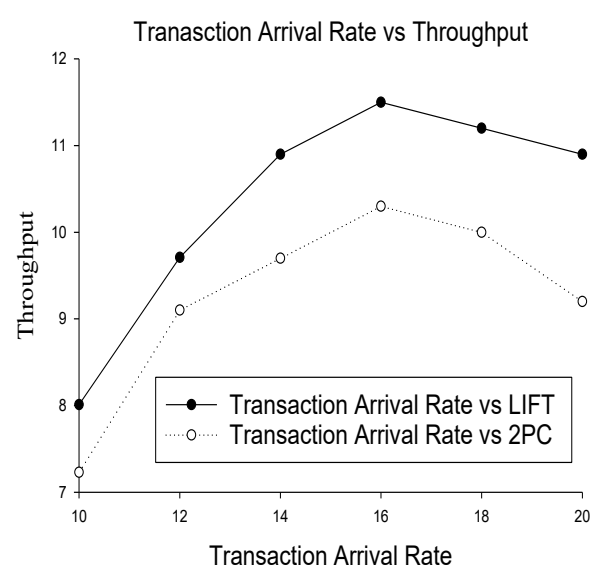

Fig 7: Comparing LIFT with 2PC using 'throughput' metric: With failure

The recovery cost associated with the $2 \mathrm{PC}$, the L$2 \mathrm{PC}$ and the proposed LIFT protocol is also compared. Since the recovery processing is about the same aside how protocols get the logs into the memory, we compared only those costs. During execution of any global transaction, logs are created in the respective disks of the sites where participating cohorts including coordinator are executing. In case of centralized 2PC, logs can be read in parallel from the various participating sites to recover a failed site that has participated in execution of global transaction. In case of L$2 \mathrm{PC}$, all the disks need to be accessed in serial order during recovery which is an inefficient and costly approach. This situation may get even worse with increase in frequency of timeouts of cohorts. With LIFT protocol, the frequency of recovery procedure is reduced by making sure that it is initiated only when required to do so. As expected, this shows better performance at higher workload.

On the whole, the proposed LIFT protocol exhibits improved performance in comparison to the $2 \mathrm{PC}$ protocol. It is largely due to the avoidance of frequent restart of commit procedure, which is ensured through an intelligent handling of timeouts using duplex communication approach among participating cohorts. 


\section{CONCLUSIONS}

With increase in distributed database based applications, having complex requirements, it is critical to redesign existing commit mechanisms. The proposed LIFT protocol is an effort in this direction. It has improved the recovery procedure of L-2PC protocol by introducing a new 'Prepare Ack' message in order to avoid restarting the entire commit processing unnecessarily. This also makes the recovery procedure more efficient in case of any such timeout of cohorts.

The performance of the above protocol is analytically evaluated considering the aspect of timeouts of cohorts. The study is just a small but decisive step in this direction and more research efforts are required to transform this into a value-added application. The concept presented in this paper deserves for the thorough attention for the future work be extended in circumstances of real-time and mobile environment [34].

\section{ACKNOWLEDGMENT}

The financial support provided by the Council of Scientific and Industrial Research (CSIR), New Delhi, India under grant no 1061461137 is acknowledged.

\section{REFERENCES}

1) Skeen, D., 1981, April. Nonblocking commit protocols. In Proceedings of the 1981 ACM SIGMOD international conference on Management of data (pp. 133-142). ACM.

2) Shanker, U., Misra, M. and Sarje, A.K., 2008. Distributed real time database systems: background and literature review. Distributed and parallel databases, 23(2), pp.127-149.

3) K. Ramamritham, "Real-time databases," Distributed and Parallel Databases, vol. 01, no. 02, pp. 199-226, 1993.

4) S. Pandey and U. Shanker, "Transaction Execution in Distributed Real-Time Database Systems," Proceedings of the International Conference on Innovations in information Embedded and Communication Systems, pp. 96-100, 2016

5) Gray, J. and Reuter, A., 1992. Transaction processing: concepts and techniques. Elsevier.

6) Gray, J.N., 1978. Notes on data base operating systems. In Operating Systems (pp. 393-481). Springer, Berlin, Heidelberg.

7) Thomas J. Watson IBM Research Center. Research Division, Lindsay, B.G., Selinger, P.G., Galtieri, C., Gray, J.N., Lorie, R.A., Price, T.G., Putzolu, F. and Wade, B.W., 1979. Notes on distributed databases.

8) Bernstein, P.A., Hadzilacos, V. and Goodman, N., 1987. Concurrency control and recovery in database systems.
9) Al-Houmaily, Y. and Chrysanthis, P., 1995, September. Two-phase commit in gigabit-networked distributed databases. In Int. Conf. on Parallel and Distributed Computing Systems (PDCS).

10) Shanker, U., Misra, M. and Sarje, A.K., 2006. SWIFTA new real time commit protocol. Distributed and Parallel Databases, 20(1), pp.29-56.

11) Al-Houmaily, Y.J., 2013. An intelligent adaptive participant's presumption protocol for atomic commitment in distributed databases. International Journal of Intelligent Information and Database Systems, 7(3), pp.242-277.

12) Al-Houmaily, Y.J., Chrysanthis, P.K. and Levitan, S.P., 1997, April. An argument in favor of the presumed commit protocol. In Data Engineering, 1997. Proceedings. 13th International Conference on (pp. 255265). IEEE.

13) Lampson, B. and Lomet, D.B., 1993, August. A new presumed commit optimization for two phase commit. In $V L D B$ (Vol. 93, pp. 630-640).

14) Lampson, B. and Lomet, D.B., Digital Equipment Corp, 1994. Distributed transaction processing using twophase commit protocol with presumed-commit without $\log$ force. U.S. Patent 5,335,343.

15) Abdallah, M., Guerraoui, R. and Pucheral, P., 1998, December. One-phase commit: does it make sense? In icpads (Vol. 98, p. 182).

16) Al-Houmaily, Y.J. and Chrysanthis, P.K., 2004, March. 1-2PC: the one-two phase atomic commit protocol. In Proceedings of the 2004 ACM symposium on Applied computing (pp. 684-691). ACM.

17) Reddy, P.K. and Kitsuregawa, M., 1998, August. Reducing the blocking in two-phase commit protocol employing backup sites. In Cooperative Information Systems, 1998. Proceedings. 3rd IFCIS International Conference on (pp. 406-415). IEEE.

18) Reddy, P.K. and Kitsuregawa, M., 2000, December. Blocking reduction in two-phase commit protocol with multiple backup sites. In International Workshop on Databases in Networked Information Systems (pp. 200215). Springer, Berlin, Heidelberg.

19) Reddy, P.K. and Kitsuregawa, M., 2003. Reducing the blocking in two-phase commit with backup sites. Information processing letters, 86(1), pp.39-47.

20) Gupta, S. and Sadoghi, M., 2018. EasyCommit: A Nonblocking Two-phase Commit Protocol. In Proceedings of the 21st international conference on extending database technology, Open Proceedings, EDBT.

21) Alkhatib, G. and Labban, R., 2002. Transaction management in distributed database systems: the case of oracle's two-phase commit. Journal of Information Systems Education, 13(2), pp.95-104. 
22) Gray, J. and Lamport, L., 2006. Consensus on transaction commit. ACM Transactions on Database Systems (TODS), 31(1), pp.133-160.

23) Pandey, S. and Shanker, U., 2018, January. Priority inversion in DRTDBS: challenges and resolutions. In Proceedings of the ACM India Joint International Conference on Data Science and Management of Data (pp. 305-309). ACM.

24) U. Shanker, Misra, M., Sarje, A.K.: Hard real-time distributed database systems: future directions. In: Proceedings of All India Seminar on Recent Trends in Computer Communication Networks. Department of ECE, IIT Roorkee, India, 7-8 November, pp. 172-177, 2001.

25) Pandey, Sarvesh, and Udai Shanker. "CART: A RealTime Concurrency Control Protocol." In Proceedings of the 22nd International Database Engineering \& Applications Symposium, pp. 119-128. ACM, 2018.

26) Pandey, Sarvesh, and Udai Shanker. "A one phase priority inheritance commit protocol." In International Conference on Distributed Computing and Internet Technology, pp. 288-294. Springer, Cham, 2018.

27) Shanker, U., Misra, M., Sarje, A.K. and Shisondia, R., 2006, December. Dependency sensitive shadow SWIFT. In 2006 10th International Database Engineering and Applications Symposium (IDEAS'06) (pp. 273-276). IEEE.
28) Shanker, U., Agarwal, N., Tiwari, S.K., Goel, P. and Srivastava, P., 2010. ACTIVE-a real time commit protocol. Wireless Sensor Network, 2(03), p.254.

29) Shanker, U., Misra, M. and Sarje, A.K., 2005, December. A memory efficient fast distributed real time commit protocol. In International Workshop on Distributed Computing (pp. 500-505). Springer, Berlin, Heidelberg.

30) Pandey, S. and Shanker, U., 2018. On Using Priority Inheritance-Based Distributed Static Two-Phase Locking Protocol. In Advances in Data and Information Sciences (pp. 179-188). Springer, Singapore.

31) S. Pandey, and U. Shanker, IDRC: A Distributed RealTime Commit Protocol. Procedia Computer Science, 125, 290 296, 2018.

32) Bernstein, P.A. and Goodman, N., 1981. Concurrency control in distributed database systems. ACM Computing Surveys (CSUR), 13(2), pp.185-221.

33) Varakantham, P.R., Gangwani, S.K. and Karlapalem, K., 2001. On handling component and transaction failures in multi agent systems. ACM SIGecom Exchanges, 3(1), pp.32-43.

34) U. Shanker, M. Misra and A. Sarje, "Some performance issues in distributed real-time database systems," Proc. VLDB Ph.D. Work,Conv. Exhib. Cent. (COEX), Seoul, Korea, 2006. 